\title{
Evaluation of confocal laser endomicroscopy for detection of occult gastric carcinoma in $\mathrm{CDH1}$ variant carriers
}

\author{
Samuel A. Schueler ${ }^{1 \#}$, Lauren A. Gamble ${ }^{2 \#}$, Bryan F. Curtin ${ }^{1}$, Samantha M. Ruff ${ }^{2}$, Maureen Connolly ${ }^{2}$, \\ Cathleen Hannah ${ }^{2}$, Martha Quezado ${ }^{3}$, Markku Miettinen ${ }^{3}$, Maureen George ${ }^{4}$, Andrew M. Blakely ${ }^{2}$, \\ Jonathan M. Hernandez ${ }^{2}$, Theo Heller ${ }^{1}$, Christopher Koh ${ }^{1}$, Jeremy L. Davis ${ }^{2}$
}

${ }^{1}$ Digestive Disease Branch, National Institute of Diabetes and Digestive and Kidney Diseases, National Institutes of Health, Bethesda, MD, USA; ${ }^{2}$ Surgical Oncology Program, Center for Cancer Research, National Cancer Institute, National Institutes of Health, Bethesda, MD, USA; ${ }^{3}$ Laboratory of Pathology, Center for Cancer Research, National Cancer Institute, National Institutes of Health, Bethesda, MD, USA; ${ }^{4}$ Clinical Center Nursing Department, National Institutes of Health, Bethesda, MD, USA

Contributions: (I) Concept and design: T Heller, C Koh, JL Davis; (II) Administrative support: M Connolly, C Hannah, M George, JL Davis; (III) Provision of study materials or patients: T Heller, C Koh, JL Davis; (IV) Collection and assembly of data: SA Schueler, LA Gamble, BF Curtin, SM Ruff, M Connolly, C Hannah, M Quezado, M Miettinen, M George, JM Hernandez, T Heller, C Koh, JL Davis; (V) Data analysis and interpretation: SA Schueler, LA Gamble, BF Curtin, SM Ruff, M Quezado, M Miettinen, AM Blakely, JM Hernandez, T Heller, C Koh, JL Davis; (VI) Manuscript writing: All authors; (VII) Final approval of manuscript: All authors.

\#These authors contributed equally to this work.

Correspondence to: Jeremy L. Davis, MD. 10 Center Drive, Room 4-3742, Bethesda, MD 20892, USA. Email: jeremy.davis@nih.gov.

Background: Hereditary diffuse gastric cancer syndrome, attributed to inactivating germline CDH1 variants, is associated with an elevated lifetime risk of gastric cancer. We sought to evaluate cancer detection using probe-based confocal laser endomicroscopy (pCLE) during endoscopic surveillance.

Methods: A prospective, single-institution study was conducted in asymptomatic adults with pathogenic or likely pathogenic (P/LP) CDH1 variants. Subjects received endoscopic gastric surveillance using pCLE in conjunction with the Cambridge method (CM). Abnormalities visualized by pCLE were biopsied, followed by non-targeted mucosal biopsies according to the CM. Primary endpoint was to determine pCLE sensitivity for detection of occult SRC carcinoma compared to CM.

Results: Thirty-six patients with P/LP CDH1 variants underwent endoscopy using pCLE and CM. Majority were female (75\%) with median age 47 years. Targeted biopsies of focal abnormalities on WLE were negative for carcinoma. Overall, 19.4\% (7/36) patients had SRC detected on $\geq 1$ biopsy. Non-targeted CM biopsies revealed SRC in 11.1\% (4/36), whereas pCLE revealed SRC in 16.7\% (6/36). Fifteen patients underwent total gastrectomy; all 15 explants contained occult carcinoma. In those 15 patients, the falsenegative SRC detection rates for pCLE and CM were $67 \%$ and $87 \%$, respectively.

Conclusions: Confocal endomicroscopy alone has low sensitivity for occult cancer detection in CDH1 variant carriers, although it appeared no worse than the current recommended method and required fewer biopsies per patient. A more reliable endoscopic surveillance is needed as a viable alternative to surgery in this high-risk population (ClinicalTrials.gov, Number: NCT03648879).

Keywords: Hereditary gastric cancer; stomach; surveillance; endoscopy

Submitted Oct 08, 2020. Accepted for publication Jan 18, 2021.

doi: 10.21037/jgo-20-430

View this article at: http://dx.doi.org/10.21037/jgo-20-430 


\section{Introduction}

Hereditary causes of gastric adenocarcinoma account for approximately $1-3 \%$ of incident cases, of which the most common is hereditary diffuse gastric cancer (HDGC) $(1,2)$. HDGC is attributed to inactivating germline variants in the CDH1 tumor suppressor gene in approximately $20-40 \%$ of cases (2-4). The CDH1 gene encodes the glycoprotein E-cadherin, which is located on the surface of epithelial cells and plays a crucial role in cell-cell adhesion. Cancer cells with metastatic potential often demonstrate loss of E-cadherin expression (5). There are more than 100 known pathogenic germline variants in the $C D H 1$ gene (2). Asymptomatic carriers of pathogenic $\mathrm{CDH} 1$ variants harbor occult foci of intramucosal signet ring cell (SRC) carcinomas in the absence of gross mucosal abnormalities, starting at a young age. The lifetime risk of developing advanced diffuse-type gastric cancer (DGC) in $\mathrm{CDH} 1$ variant carriers is $33-42 \%$ based on revised penetrance estimates $(6,7)$. Prophylactic total gastrectomy is recommended in variant carriers starting as early as 20 years of age $(2,4)$. However, total gastrectomy is associated with life-long morbidity that includes major weight loss and micronutrient deficiencies $(8,9)$.

An alternative to prophylactic surgery is regular endoscopic surveillance of the gastric mucosa. For patients who choose to delay or forego total gastrectomy, annual high-definition white light endoscopy (WLE) with six biopsies from each anatomical zone of the stomach (antrum, transitional zone, body, fundus, and cardia) and any other visible lesions is recommended, and is referred to as the Cambridge method (CM) $(4,10)$. However, endoscopic surveillance with random mucosal biopsies in these patients carries an unacceptably high (up to $96 \%$ ) false-negative rate (11-13). For reference, occult, in situ or early-stage (T1a) SRC carcinomas are found in up to $100 \%$ of gastrectomy explants from asymptomatic carriers of $C D H 1$ pathogenic variants $(12,13)$. The false negative rate of random endoscopic biopsy is attributed to the large surface area of gastric mucosa and the typical sub-epithelial location of signet ring cancer cells.

Probe-based confocal laser endomicroscopy (pCLE) enables endoscopists to obtain in vivo histologic images of gastrointestinal mucosa $(14,15)$. A fiberoptic probe inserted through a standard endoscope is used to image the microstructure of mucosal tissues including, but not limited to, the identification of cells and vessels and their organization or architecture. Until now, pCLE has not been evaluated as a surveillance modality in patients with HDGC due to pathogenic germline $C D H 1$ variants. We propose this technique may afford a more sensitive method of surveillance of occult gastric cancer in asymptomatic carriers of pathogenic $C D H 1$ variants. We conducted a clinical trial to determine if pCLE provides greater sensitivity for detection of SRC foci compared to the Cambridge method, and to define the false negative rate of pCLE cancer detection in patients who subsequently choose prophylactic total gastrectomy.

We present the study in accordance with the MDAR reporting checklist (available at http://dx.doi.org/10.21037/ jgo-20-430).

\section{Methods}

The study was conducted in accordance with the Declaration of Helsinki (as revised in 2013). The study was approved by the institutional review board of the National Institutes of Health (reference number 385481) and informed consent was taken from all patients.

\section{Study population}

A single-arm, phase II clinical trial was open to patient enrollment from February 2019 through October 2019. Eligible patients were asymptomatic carriers of pathogenic or likely pathogenic (P/LP) CDH1 variants, 18 years or older, physiologically able to undergo upper endoscopy, and able to provide written informed consent. Patients with concurrent illness that would preclude upper gastrointestinal endoscopy were excluded; specifically, unstable angina, recent (within 3 months) myocardial infarction, contraindications to general anesthesia, and known bleeding disorders.

\section{Study procedures}

All patients underwent high definition WLE with a $2 \mathrm{~T}$ endoscope to facilitate pCLE and cold forceps biopsy nearly simultaneously (Olympus GIF-2TH180 endoscope, Global Endoscopy Solutions). All patients were administered monitored anesthesia care or general anesthesia. Examinations began with digital image capture under WLE from 22 areas of the stomach using a previously described systematic mapping schema (16). These areas include antegrade 4-section views (anterior wall, lesser 
curve, posterior wall, and greater curve) of the antrum, lower body, and middle upper body followed by retrograde 4 -section views of the fundus and retrograde 3 -section views (anterior wall, lesser curve, posterior wall) of incisura, middle upper body and cardia/fundus (Figure S1). Probebased CLE was performed before any biopsy of focal abnormality, but only after complete WLE examination and image capture of all twenty-two areas. Next, intravenous fluorescein $(250 \mathrm{mg} ; 2.5 \mathrm{~mL}$ of $100 \mathrm{mg} / \mathrm{mL}$ solution) was administered followed by $10 \mathrm{~mL}$ saline flush. Probe-based CLE evaluation was performed immediately following fluorescein injection $\left(\right.$ Cellvizio ${ }^{\circledR} 100$ series systems with Confocal Miniprobes ${ }^{\mathrm{TM}}$, Mauna Kea Technologies). Two study investigators were present and conducted real-time confocal analysis of each of the 22 aforementioned areas of the stomach by extending the probe onto the surface of normal-appearing gastric mucosa. Digital video and still image capture were performed to document both regular and irregular confocal findings from each section. Bite-onbite gastric biopsies were obtained during the confocal exam with agreement by the investigators. Cold biopsy forceps with radial jaw and needle was used (Boston Scientific, $2.8 \mathrm{~mm}$ ). After conclusion of pCLE evaluation, nontargeted gastric biopsies were obtained according to the Cambridge method; 6 biopsies each from antrum, transitional zone, body, cardia and fundus (10). Abnormal findings via WLE, such as pale mucosal areas, were also recorded and/or biopsied separately during the procedure, but only after pCLE evaluation to avoid image interference from mucosal disruption. Images obtained by pCLE and WLE images were annotated in conjunction with tissue biopsy location data.

\section{Specimen processing}

All samples were placed in formalin and labeled according to anatomic location. Biopsy tissue was processed through cycles of formalin, alcohol, xylene, and paraffin blocks cut 4-microns thick, which were then stained with hematoxylin and eosin. All biopsies were examined by two experienced gastrointestinal pathologists (MM, MQ) blinded to clinical endoscopic findings and source of biopsies (e.g., Cambridge or pCLE). All biopsies and resection specimens underwent staining with hematoxylin and eosin. Periodic acid-Schiff staining was performed in all biopsies and selected sections of resection specimens to facilitate identification of signet ring cells. Biopsies revealing occult carcinomas were reviewed by the principal investigator after pathologic consensus diagnosis. All patients were notified of biopsy findings and received appropriate counseling regarding gastric cancer risk.

\section{Statistical analysis}

Based on our institutional occult cancer detection rate of $24 \%$ with surveillance endoscopy, 36 patients undergoing pCLE would give $89 \%$ power to rule out a $24 \%$ rate of detection in favor of a $45 \%$ rate of detection with a one-sided 0.10 significance level exact binomial test. An accrual ceiling of 40 was set to allow for a small number of unevaluable patients. Standard analysis was performed on the two tests (pCLE and CM) including sensitivity with a paired-samples analysis, false negative rates, and diagnostic accuracy was estimated using $95 \%$ confidence interval. The paired-samples analysis for sensitivity was obtained from the histopathology of specimen samples following total gastrectomy. Fisher exact test was used to compare the biopsy positivity rate of pCLE and CM. All authors had access to the study data and reviewed and approved the final manuscript.

\section{Results}

\section{Patient population}

Thirty-seven patients with $C D H 1$ variants underwent WLE and pCLE examination per protocol. Thirty-six patients harbored a $\mathrm{P} / \mathrm{LP} C D H 1$ variant and are the subject of analysis (Table 1). One patient who underwent pCLE was later determined to carry a $C D H 1$ variant of uncertain significance and was removed from study. The majority $(75 \%)$ of patients were female, $89 \%$ were Caucasian, median age was 47 years (range 25-74), and 61\% had previously undergone upper endoscopy. Median procedure time, inclusive of both pCLE and CM evaluations, was 52.5 minutes. Less than half the study population $(36.1 \%)$ met consensus criteria for HDGC genetic testing. Pathogenic or likely pathogenic $C D H 1$ variants were: 9 nonsense, 8 frameshift, 7 cryptic splice, 5 canonical splice, 4 intronic splicing, and 3 deletion (Table 1).

\section{WLE endoscopy findings}

Standard WLE endoscopy identified mucosal abnormalities in $30 / 36(83.3 \%)$ patients, including erythematous, 
Table 1 Patient demographics and pCLE procedure characteristics

\begin{tabular}{|c|c|}
\hline Characteristic & Value \\
\hline Age in years, median (IQR) & $47(37.5,61.25)$ \\
\hline \multicolumn{2}{|l|}{ Sex (\%) } \\
\hline Male & $9(25)$ \\
\hline Female & $27(75)$ \\
\hline \multicolumn{2}{|l|}{ Race (\%) } \\
\hline Caucasian & $34(94)$ \\
\hline African American & $1(3)$ \\
\hline Hispanic & $1(3)$ \\
\hline Procedure time (minutes), median (IQR) & $52.5(44,64.5)$ \\
\hline pCLE biopsies per patient, median (IQR) & $7.5(6,9)$ \\
\hline $\begin{array}{l}\text { Cambridge method biopsies per patient } \\
\text { (IQR) }\end{array}$ & $30(30,30)$ \\
\hline Patients with targeted WLE biopsies (\%) & $19(52.8)$ \\
\hline \multicolumn{2}{|l|}{$\mathrm{CDH} 1$ Variant } \\
\hline Pathogenic (\%) & $25(69)$ \\
\hline Likely pathogenic (\%) & $11(31)$ \\
\hline Nonsense (\%) & $9(25)$ \\
\hline c. $172 \mathrm{G}>\mathrm{T}$ & $3(8)$ \\
\hline c. $1792 \mathrm{C}>\mathrm{T}$ & $2(6)$ \\
\hline c.2064_2065del & $3(9)$ \\
\hline c.2064_2065delTG & $1(3)$ \\
\hline Frameshift (\%) & $8(22)$ \\
\hline c.603delT & $2(6)$ \\
\hline c. $1145 \mathrm{del}$ & $1(3)$ \\
\hline c.1460_1461delTG & $1(3)$ \\
\hline c.1779dupC & $1(3)$ \\
\hline c.1982delG & $1(3)$ \\
\hline c.2430del & 1 (3) \\
\hline c.720delT & $1(3)$ \\
\hline Cryptic splice (\%) & 7 (19) \\
\hline c. $2195 \mathrm{G}>\mathrm{A}$ & $5(14)$ \\
\hline c. $715 \mathrm{G}>\mathrm{A}$ & $2(6)$ \\
\hline
\end{tabular}

Table 1 (continued)
Table 1 (continued)

\begin{tabular}{lc}
\hline Characteristic & Value \\
\hline Canonical splice (\%) & $5(14)$ \\
c.1565+1G>T & $2(6)$ \\
c.1565+1G>C & $1(3)$ \\
c.49-2A>C & $1(3)$ \\
c.833-2A>G & $1(3)$ \\
Intronic splicing (\%) & $4(11)$ \\
c.2439+5_2439+8delGTAA & $3(8)$ \\
c.532-1G>C & $1(3)$ \\
Deletion (\%) & $3(8)$ \\
Deletion exon 3 & $2(6)$ \\
Deletion exons 1-2 & $1(3)$ \\
\hline
\end{tabular}

erosive, or ulcerated mucosa $(12 / 36,33.3 \%)$, gastric polyps $(11 / 36,30.1 \%)$, gastric nodule(s)/papule(s) or nodularappearing mucosa $(9 / 36,25 \%)$, mucosal atrophy $(4 / 36$, $11.1 \%)$, friable mucosa $(4 / 36,11.1 \%)$, or white spots $(2 / 36,5.6 \%)$. Nineteen of $36(52.8 \%)$ patients underwent either 1 or 2 mucosal biopsies due to WLE findings. Subsequent pathologic analysis of these targeted biopsies revealed gastritis in $8 / 36(22.2 \%)$ and fundic gland polyps in $4 / 36(11.1 \%)$. One patient had biopsy evidence of Helicobacter pylori infection. WLE with targeted biopsies failed to identify any occult SRC carcinoma (Table S1). The remaining six patients $(16.7 \%)$ had normal-appearing gastric mucosa on WLE examination.

\section{Surveillance biopsy results}

Overall, 7 of 36 (19.4\%) patients had occult SRC foci discovered on thirteen separate biopsies during surveillance endoscopy. Eleven of those $13(84.6 \%)$ positive biopsies were obtained from the gastric fundus. In total, 282 pCLEguided biopsies were obtained with a median of 7.8 per patient (IQR 6, 9). Six patients $(16.7 \%, 6 / 36)$ underwent pCLE-directed gastric biopsy that contained occult SRC carcinoma. This consisted of nine separate pCLE biopsies, which revealed a cancer focus of median size $0.5 \mathrm{~mm}$ (IQR $0.25,1.25)$ in the following anatomic locations: posterior 
A

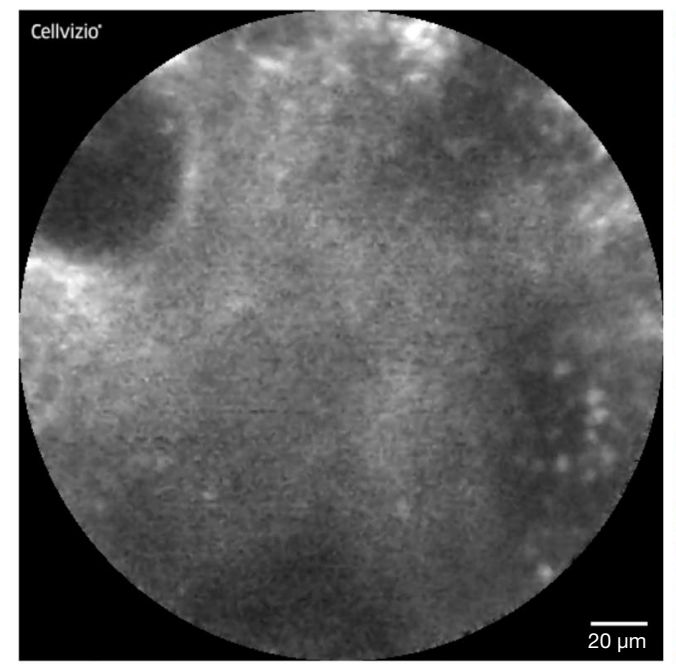

C

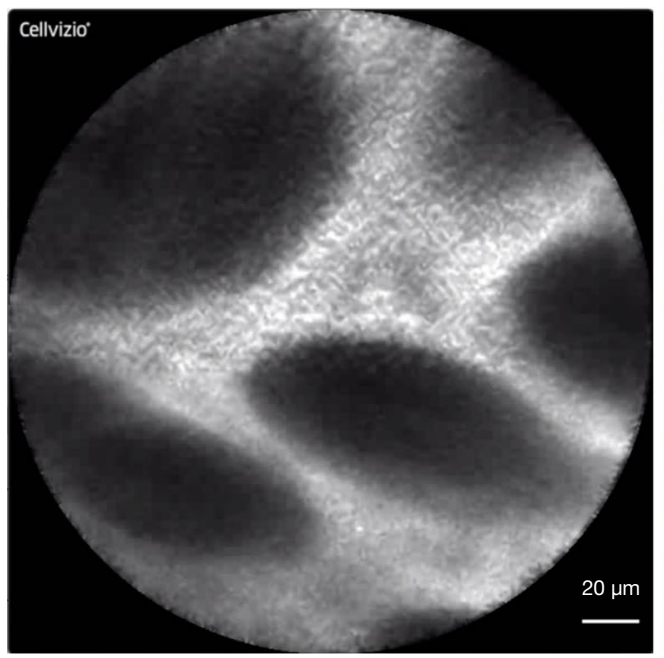

B

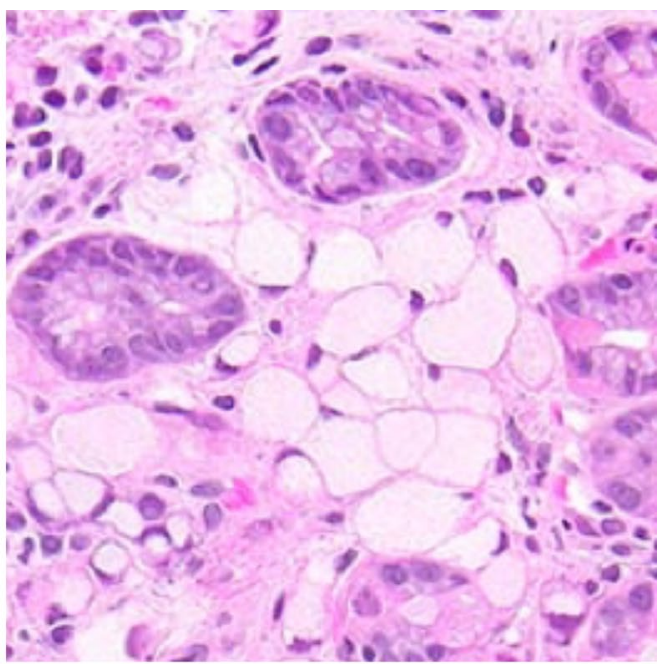

$\mathrm{D}$

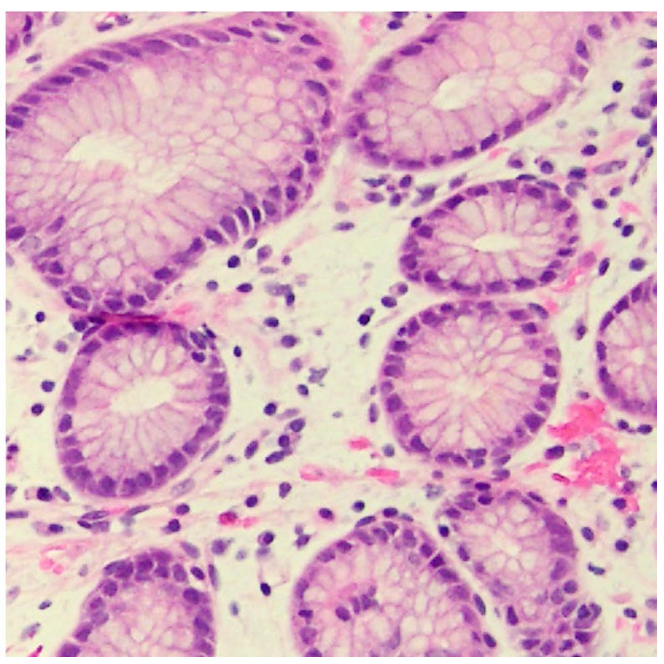

Figure 1 Atypical pCLE images of gastric mucosal pathology with matched biopsy photomicrographs. Abnormal appearing confocal laser endomicroscopic image (A) with cross-sectional view of gastric pits displaced by the expansion of the lamina propria by signet ring cells and (B) corresponding biopsy (hematoxylin and eosin, 40×). Abnormal appearing confocal laser endomicroscopic image (C) with cross-sectional view of gastric pits with minimal displacement by stromal edema within lamina propria (D) and corresponding (4 biopsies), anterior fundus.

fundus (4 biopsies), anterior fundus (3), posterior upper middle body (1), and retroflex view lesser upper middle body (1). Abnormal pCLE images with corresponding biopsy photomicrographs (Figure 1) illustrate the irregular appearance of displaced gastric pits, which was not specific for cancer. Typical pCLE images of gastric mucosa (Figure 2) demonstrated no pathologic changes on biopsy.

Cambridge method of non-targeted biopsies was performed in all patients following pCLE examination.
A total of 1,080 CM biopsies were obtained. Occult SRC carcinoma was detected in 4 patients, all from the gastric fundus. The overall detection of occult cancer using CM of non-targeted biopsies was $11.1 \%$ (4/36). CM detected SRC carcinoma in 3 of the 6 patients for which pCLE also detected cancer foci. pCLE biopsies detected SRC carcinoma in 3 of the 4 patients for which CM also detected cancer foci. According to surveillance biopsy method, the overall positive biopsy rate was $2.1 \%(6 / 282)$ with pCLE 
A

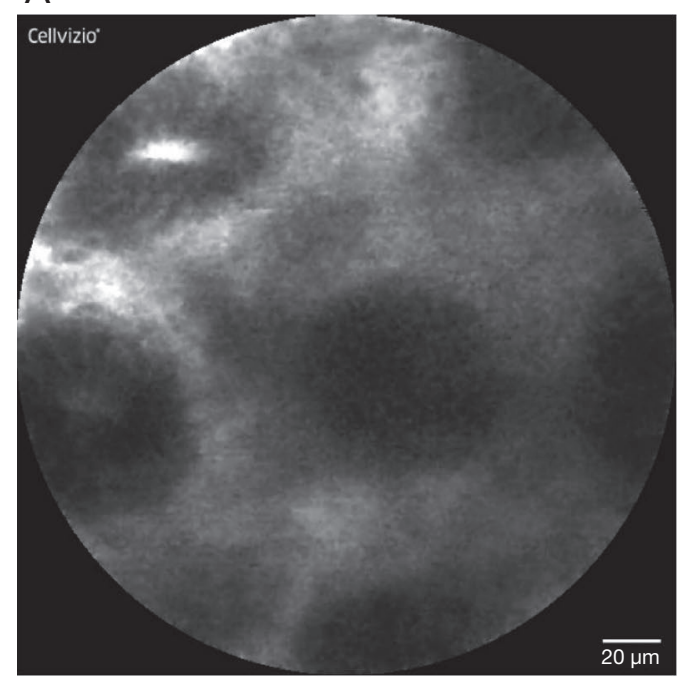

C

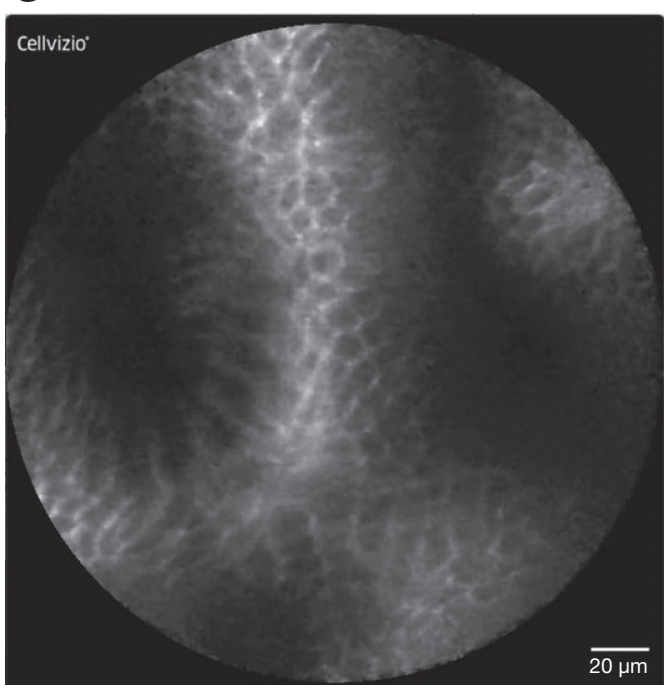

B

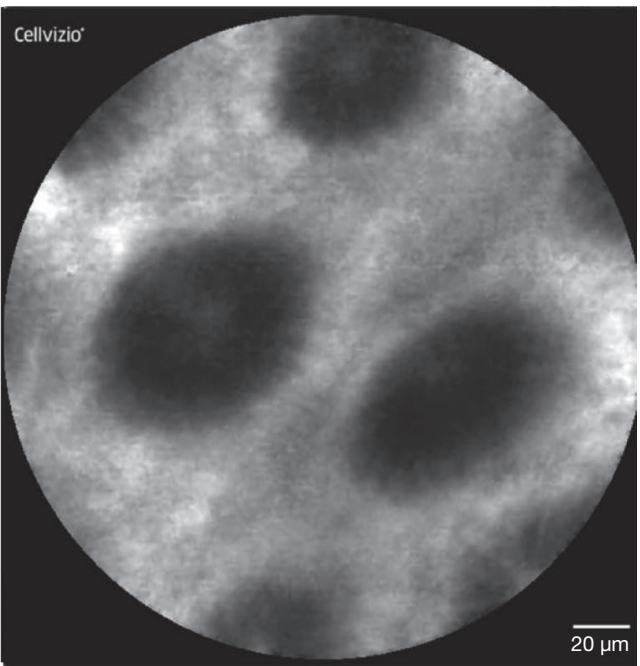

D

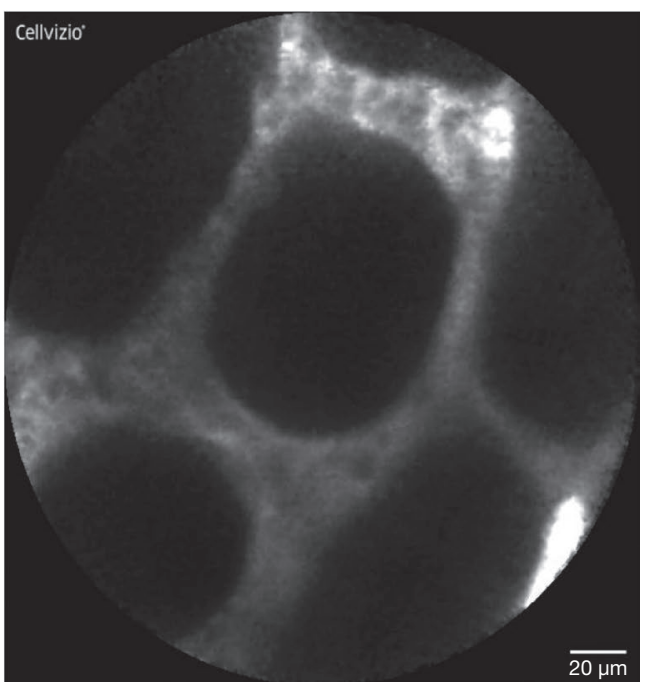

Figure 2 Probe-based CLE images of gastric mucosa interpreted as normal and confirmed by biopsy with histopathology. Normal appearing confocal laser endomicroscopic image of gastric fundus (A), body (B), antrum (C), and incisura (D).

and $0.4 \%(4 / 1,080)$ with $\mathrm{CM}(\mathrm{P}<0.05)$. At 14 days postprocedure there were no serious adverse events related to pCLE, fluorescein administration, or upper gastrointestinal endoscopy. During one pCLE examination the probe cap detached within the gastric body and was safely retrieved without sequelae.

\section{True cancer rates}

Fifteen patients (41.6\%) subsequently elected for prophylactic total gastrectomy. All 15 gastrectomy specimens contained SRC carcinomas (T1aN0) and the average size of SRC foci was $1.9 \mathrm{~mm}$ (range, 0.1-8.0 mm). There was no difference in cancer detection rates based on $C D H 1$ variant type by endoscopic biopsy or total gastrectomy. In those 15 patients, pCLE detected SRC in 3 patients that were missed by CM, while there were no instances of SRC found by CM that were not found by pCLE. Confocal laser endomicroscopy detected occult SRC in five patients yielding a sensitivity rate of $33.3 \%(5 / 15)$, whereas CM detected SRC in 2 resulting in a sensitivity of only $13.3 \%(2 / 15)$. The false negative detection rates for pCLE and CM were $66.7 \%(10 / 15)$ and $86.7 \%(13 / 15)$, respectively. Most SRC foci detected endoscopically were 
from biopsies obtained from the fundus $(84.6 \%, 11 / 13)$ while pCLE biopsies detected an additional 2 foci of SRC in the gastric body. Similarly, upon total gastrectomy explant analysis, cancer foci were reported in the cardia/fundus and body, but not at the incisura or antrum.

\section{Discussion}

In this single-institution phase II study we demonstrated that pCLE could serve as an adjunct to standard endoscopy to improve occult cancer detection in $C D H 1$ variant carriers. Probe-based CLE biopsies demonstrated a lower false negative rate of occult cancer detection when compared to both CM biopsy and WLE targeted biopsy of mucosal abnormalities. Although international consensus guidelines recommend prophylactic total gastrectomy for patients with pathogenic $C D H 1$ variants, many choose to delay or never pursue this procedure, while others often decide to pursue gastrectomy only after cancer foci have been identified on endoscopic biopsy. Because of this, patients and clinicians alike desire a reliable method of endoscopic cancer detection, and in some cases surveillance of occult cancer foci, yet current endoscopic techniques remain insensitive.

This is the first prospective clinical trial of endoscopic surveillance for cancer in $C D H 1$ variant carriers (11,17-19). Using the currently recommended CM, the overall rate of occult carcinoma detection in this study was $19 \%$. By comparison, Mi et al. reported an overall $61 \%$ SRC detection rate in $C D H 1$ pathogenic variant carriers at an expert center. However, based on initial (single) endoscopy, their reported detection rate was $39 \%$, which is similar to rates of $40-50 \%$ described by others $(18,20)$. Even so, the rates of occult gastric cancer detection using the Cambridge method are not consistent across centers. Two recent reports of surveillance endoscopy using $\mathrm{CM}$ describe lower cancer detection rates of $14.6 \%(7 / 48)(17)$ and $12 \%(3 / 25)$ (21). Moreover, our endoscopists (TH, CK) conduct surveillance endoscopies weekly for $\mathrm{CDH} 1$ variant carriers and patients with the hereditary diffuse gastric cancer syndrome. In our experience, occult SRC detection with endoscopic surveillance of asymptomatic patients has ranged from 15 to $36 \%$ (unpublished data) (16). However, the individual cancer detection rates using pCLE (16.7\%) or CM (11.1\%) are still lower than expected. We did not observe any differences in genotype, sex, race, or ethnicity that would explain low detection rates.

Adjuncts to WLE have been evaluated in an attempt to improve sensitivity of early gastric cancer detection in this population. For instance, NBI in tandem with WLE has proven helpful for delineating pale areas and has a high negative predictive value for abnormal-appearing vascular and mucosal $(11,22)$. However, pale areas were rarely seen in the current study and are known to be non-specific for SRC foci (22). This lack of specificity has been validated in other studies that demonstrated non-elevated pale lesions revealed SRC histology in only $17 \%$ of biopsies, whereas the majority revealed no histologic change or simply inflammation (20). Targeted biopsy of any focal abnormality in their report demonstrated SRC in only $11 \%$ of samples. It follows that occult SRC cancers discovered in our study, both by pCLE and CM, were obtained from grossly normal gastric mucosa, without pale spots or protruding lesions, which is consistent with other reports $(18,20)$. Another technique, chromoendoscopy, may improve sensitivity by illuminating pale white spots of gastric mucosa and other suspicious lesions, however this technique is reportedly unable to detect SRC foci less than $4 \mathrm{~mm}$ in size (23). Notably, in our study, the typical size of SRC foci detected on endoscopic biopsy was considerably less than $1 \mathrm{~mm}$. This fact alone reinforces the purported limitation of chromoendoscopy for detection of submillimeter SRC foci, which are pathognomonic of HDGC. The addition of endoscopic ultrasound (EUS) was also evaluated in a series of asymptomatic $C D H 1$ variant carriers but did not improve sensitivity (24). It is still worth considering that a combination of endoscopic modalities may hold the potential to improve early detection.

Although we hypothesize that the pCLE pattern of displaced gastric pits may help identify SRC foci, further investigation is needed to rule out the possibility that pCLE biopsies captured SRC by chance. However, we did not detect any SRC foci by WLE-targeted biopsy alone. This may be due to the focused nature of this study, with fewer biopsies taken of incidental abnormal findings (e.g., atrophic gastric mucosa). Comparison of pCLE images and videos compared with corresponding biopsies, potentially aided by machine learning, could help to further identify endoscopic pCLE patterns indicative of SRC involvement and is an area of active research by the authors. Interestingly, the gastric fundus was the most common site overall for biopsies that revealed SRC foci $(11 / 13,84.6 \%)$, which held true also for the majority of pCLE-based biopsies (7/9, 77.8\%). Comparatively, $45.2 \%$ of all SRC foci detected by Mi et al. were in the fundus or cardia (11). Despite these findings, an anatomic predisposition to SRC development within the 
stomach, or genotype-phenotype correlations of such, has yet to be determined.

Among patients who underwent risk-reducing total gastrectomy, the false negative rate of SRC detection by pCLE was substantially lower than that of the Cambridge method. Probe-based CLE detected more SRC foci than $\mathrm{CM}$ in this subset of patients, with an average of twentytwo fewer biopsies taken per patient. Our finding that early stage gastric cancer was detected in 15/15 (100\%) gastrectomy specimens is at the extreme of SRC detection at total gastrectomy from other studies, ranging from $87 \%$ to $100 \%(12,13,22,25,26)$. A recent review reinforced the challenge of false-negative biopsies by reporting that endoscopic surveillance can miss $45-60 \%$ of SRC foci ultimately present in gastrectomy specimens (27). It remains unknown, however, whether disease penetrance differs by $C D H 1$ genotype and if this may also have an impact on early carcinogenesis. The fact that nearly all patients who undergo prophylactic total gastrectomy harbor early gastric carcinomas, and development of advanced gastric cancer is not absolute, suggests more research is needed to understand the spectrum of this disease.

Clinical trials for this rare cancer syndrome are scarce and therefore warrant critical appraisal and acknowledgment of limitations. This prospective study of individuals harboring a rare genetic variant was conducted over a short time period by a consistent group of investigators, which should lessen the variability in practice that may be more common in retrospective studies of this patient population. A limitation of this study is the singlearm design, which reduced our ability to fully distinguish pCLE from CM, even though an intra-patient comparison of pCLE and CM was performed. A practical criticism is the added endoscopy time for pCLE and thus the potential inability to generalize to standard medical practice. In addition, mucolytics, simethicone, and acid suppressing medications, which may have improved mucosal visibility and pCLE imaging, were not used. Additionally, the limited working depth of examination could have excluded the presence of SRC deeper within the lamina propria. Confocal image interpretation with objective criteria were preferred, however no such criteria exist. Posthoc image quantification was performed using a machine learning algorithm, however this was exploratory and not a pre-specified study endpoint. Despite these limitations, eligibility criteria were broad, and enrollment occurred contemporaneously with clinical care in an attempt to avoid selection bias. Operator bias was mitigated by performing pCLE prior to CM such that any abnormalities induced by biopsy or hemorrhage would be reduced. We contend that use of a $2 \mathrm{~T}$ endoscope maximized efficiency and accuracy in obtaining mucosal biopsies with pCLE, however its use may also be described as impractical. While the study was powered purposefully to detect a large, and clinically meaningful difference in cancer detection, a larger study population would have allowed more observations to inform our appraisal of pCLE as a potential tool for gastric cancer surveillance.

In conclusion, pCLE performed with white light surveillance endoscopy has a low rate of detection of occult gastric carcinoma in $\mathrm{CDH} 1$ variant carriers. Ideally, the aim of cancer surveillance in this population should be the detection of clinically relevant cancer foci that harbor true potential for progression to advanced gastric cancer. However, our ability both to detect these early cancers and predict their biologic behavior is lacking. Endoscopic surveillance will continue to provide only a binary outcome that is imperfect at best and unreliable at its worst. Clinical management including endoscopic surveillance as an alternative to surgery should be tempered by this reality. Further exploration of advanced endoscopic techniques, including confocal endomicroscopy perhaps augmented by artificial intelligence, is warranted.

\section{Acknowledgments}

Funding: This study was supported in part by the Intramural Research Program, National Cancer Institute, National Institutes of Health.

\section{Footnote}

Reporting Checklist: The authors have completed the MDAR reporting checklist. Available at http://dx.doi.org/10.21037/ jgo-20-430

Peer Review File: Available at http://dx.doi.org/10.21037/ jgo-20-430

Data Sharing Statement: Available at http://dx.doi. org/10.21037/jgo-20-430

Conflicts of Interest: All authors have completed the ICMJE uniform disclosure form (available at: http://dx.doi. org/10.21037/jgo-20-430). The authors have no conflicts of 
interest to declare.

Ethical Statement: The authors are accountable for all aspects of the work in ensuring that questions related to the accuracy or integrity of any part of the work are appropriately investigated and resolved. The study was conducted in accordance with the Declaration of Helsinki (as revised in 2013). The study was approved by the institutional review board of the National Institutes of Health (reference number 385481) and informed consent was taken from all patients.

Open Access Statement: This is an Open Access article distributed in accordance with the Creative Commons Attribution-NonCommercial-NoDerivs 4.0 International License (CC BY-NC-ND 4.0), which permits the noncommercial replication and distribution of the article with the strict proviso that no changes or edits are made and the original work is properly cited (including links to both the formal publication through the relevant DOI and the license). See: https://creativecommons.org/licenses/by-nc-nd/4.0/.

\section{References}

1. Bray F, Ferlay J, Soerjomataram I, et al. Global cancer statistics 2018: GLOBOCAN estimates of incidence and mortality worldwide for 36 cancers in 185 countries. CA Cancer J Clin 2018;68:394-424.

2. Hansford S, Kaurah P, Li-Chang H, et al. Hereditary Diffuse Gastric Cancer Syndrome: CDH1 Mutations and Beyond. JAMA Oncol 2015;1:23-32.

3. Guilford P, Hopkins J, Harraway J, et al. E-cadherin germline mutations in familial gastric cancer. Nature 1998;392:402-5.

4. Blair VR, McLeod M, Carneiro F, et al. Hereditary diffuse gastric cancer: updated clinical practice guidelines. Lancet Oncol 2020;21:e386-97.

5. Pecina-Slaus N. Tumor suppressor gene E-cadherin and its role in normal and malignant cells. Cancer Cell Int 2003;3:17.

6. Xicola RM, Li S, Rodriguez N, et al. Clinical features and cancer risk in families with pathogenic CDH1 variants irrespective of clinical criteria. J Med Genet 2019;56:838-43.

7. Roberts ME, Ranola JMO, Marshall ML, et al. Comparison of CDH1 Penetrance Estimates in Clinically Ascertained Families vs Families Ascertained for Multiple Gastric Cancers. JAMA Oncol 2019;5:1325-31.
8. Liedman B. Symptoms after total gastrectomy on food intake, body composition, bone metabolism, and quality of life in gastric cancer patients--is reconstruction with a reservoir worthwhile? Nutrition 1999;15:677-82.

9. Lewis FR, Mellinger JD, Hayashi A, et al. Prophylactic total gastrectomy for familial gastric cancer. Surgery 2001;130:612-7; discussion 617-9.

10. Fitzgerald RC, Hardwick R, Huntsman D, et al. Hereditary diffuse gastric cancer: updated consensus guidelines for clinical management and directions for future research. J Med Genet 2010;47:436-44.

11. Mi EZ, Mi EZ, di Pietro M, et al. Comparative study of endoscopic surveillance in hereditary diffuse gastric cancer according to CDH1 mutation status. Gastrointest Endosc 2018;87:408-18.

12. Norton JA, Ham CM, Van Dam J, et al. CDH1 truncating mutations in the E-cadherin gene: an indication for total gastrectomy to treat hereditary diffuse gastric cancer. Ann Surg 2007;245:873-9.

13. Rogers WM, Dobo E, Norton JA, et al. Risk-reducing total gastrectomy for germline mutations in E-cadherin (CDH1): pathologic findings with clinical implications. Am J Surg Pathol 2008;32:799-809.

14. Kitabatake S, Niwa Y, Miyahara R, et al. Confocal endomicroscopy for the diagnosis of gastric cancer in vivo. Endoscopy 2006;38:1110-4.

15. Kakeji Y, Yamaguchi S, Yoshida D, et al. Development and assessment of morphologic criteria for diagnosing gastric cancer using confocal endomicroscopy: an ex vivo and in vivo study. Endoscopy 2006;38:886-90.

16. Curtin BF, Hernandez JM, Quezado M, et al. Systematic Screening Protocol for the Stomach (Ssp) Is Superior to Standard Endoscopy for the Detection of Early Malignancy in Hereditary Gastric Cancer Syndrome Patients. Gastroenterology 2019;156:S22.

17. Friedman M, Adar T, Patel D, et al. Surveillance Endoscopy in the Management of Hereditary Diffuse Gastric Cancer Syndrome. Clin Gastroenterol Hepatol 2021;19:189-91.

18. Jacobs MF, Dust H, Koeppe E, et al. Outcomes of Endoscopic Surveillance in Individuals With Genetic Predisposition to Hereditary Diffuse Gastric Cancer. Gastroenterology 2019;157:87-96.

19. Van Dieren JM, Kodach LL, Cats A. Targeted Versus Random Biopsies in Surveillance Endoscopy in Hereditary Diffuse Gastric Cancer Syndrome. Clin Gastroenterol Hepatol 2020;18:1647-8.

20. van Dieren JM, Kodach LL, den Hartog P, et al. 
Gastroscopic surveillance with targeted biopsies compared with random biopsies in CDH1 mutation carriers.

Endoscopy 2020;52:839-46.

21. Castro R, Lobo J, Pita I, et al. Random biopsies in patients harboring a CDH1 mutation: time to change the approach? Rev Esp Enferm Dig 2020;112:367-72.

22. Lim YC, di Pietro M, O'Donovan M, et al. Prospective cohort study assessing outcomes of patients from families fulfilling criteria for hereditary diffuse gastric cancer undergoing endoscopic surveillance. Gastrointest Endosc 2014;80:78-87.

23. Shaw D, Blair V, Framp A, et al. Chromoendoscopic surveillance in hereditary diffuse gastric cancer: an alternative to prophylactic gastrectomy? Gut 2005;54:461-8.

Cite this article as: Schueler SA, Gamble LA, Curtin BF, Ruff SM, Connolly M, Hannah C, Quezado M, Miettinen M, George M, Blakely AM, Hernandez JM, Heller T, Koh C, Davis JL. Evaluation of confocal laser endomicroscopy for detection of occult gastric carcinoma in $C D H 1$ variant carriers. J Gastrointest Oncol 2021;12(2):216-225. doi: 10.21037/jgo-20-430
24. Kumar S, Katona BW, Long JM, et al. Endoscopic Ultrasound Has Limited Utility in Diagnosis of Gastric Cancer in Carriers of CDH1 Mutations. Clin Gastroenterol Hepatol 2020;18:505-8.e1.

25. Seevaratnam R, Coburn N, Cardoso R, et al. A systematic review of the indications for genetic testing and prophylactic gastrectomy among patients with hereditary diffuse gastric cancer. Gastric Cancer 2012;15 Suppl 1:S153-63.

26. Chen Y, Kingham K, Ford JM, et al. A prospective study of total gastrectomy for CDH1-positive hereditary diffuse gastric cancer. Ann Surg Oncol 2011;18:2594-8.

27. Kumar S, Long JM, Ginsberg GG, et al. The role of endoscopy in the management of hereditary diffuse gastric cancer syndrome. World J Gastroenterol 2019;25:2878-86. 


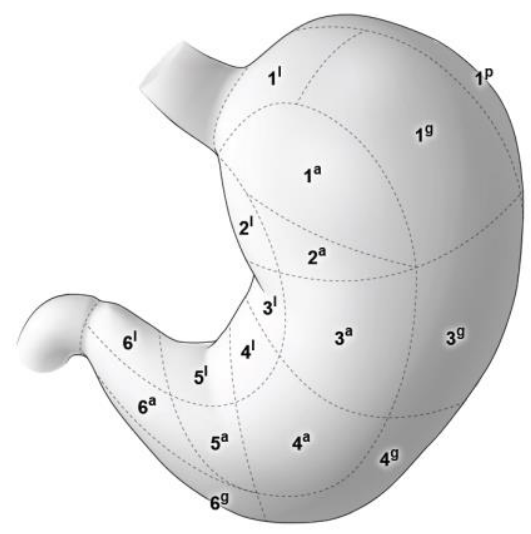

Anterior view

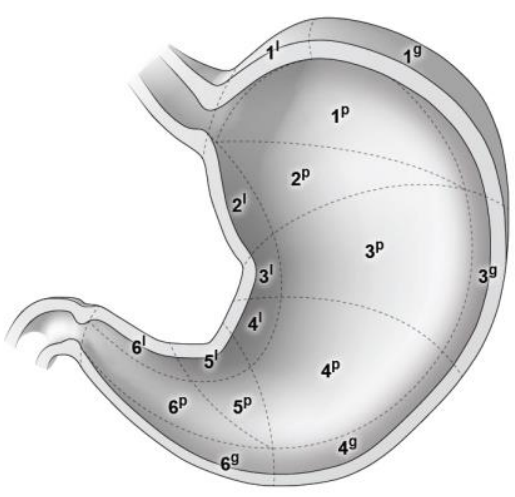

Posterior view

Figure S1 Map of twenty-two anatomic zones for probe-based confocal endomicroscopic evaluation of gastric mucosa.

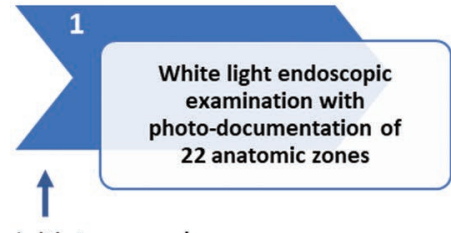

Initiate procedure

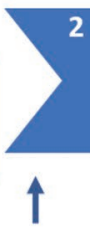

Fluorescein injection

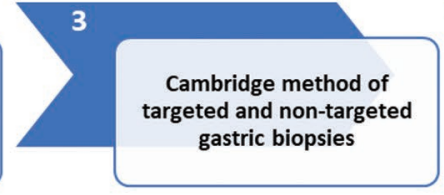

eted and non-targeted

pCLE examination of each of 22 zones with/without gastric biopsy

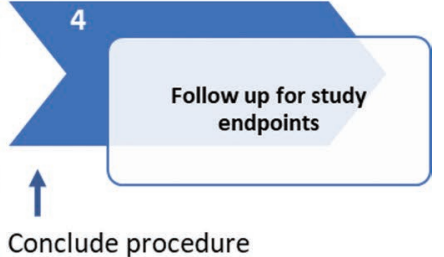

Conclude procedure

Figure S2 Schematic representation of protocol-based intervention. 


\begin{tabular}{|c|c|c|c|c|c|c|}
\hline \multirow{2}{*}{ Patient } & \multicolumn{2}{|l|}{ While light endoscopy } & \multicolumn{2}{|l|}{ Probe-based CLE } & \multirow{2}{*}{$\begin{array}{l}\text { Cambridge method } \\
\text { SRC location/size (mm) }\end{array}$} & \multirow{2}{*}{$\begin{array}{l}\text { Gastrectomy explant } \\
\text { SRC location/size (largest focus, mm) }\end{array}$} \\
\hline & Visual findings & Biopsy results & Number of biopsies & SRC Iocation/size (mm) & & \\
\hline 1 & Mucosal atrophy & n/a & 7 & n/a & Fundus/1.0 & $n / a$ \\
\hline 2 & Polyps & Chronic gastritis & 7 & n/a & $n / a$ & Fundus/8.0 \\
\hline 3 & Retained endoclip; multiple small white scars & $n / a$ & 9 & $n / a$ & $n / a$ & Upper fundus/1.1 \\
\hline 4 & Polyps & Gastric mucosa with occasional dilated glands & 4 & Posterior fundus/1.7 & Fundus/ 0.7 & Fundus $/ 2.0$ \\
\hline 5 & Mucosal atrophy, polyps & $n / a$ & 9 & Posterior fundus/1.6 & Fundus/0.5 & Upper fundus/3.0 \\
\hline 6 & Eythematous and ulcerated antral mucosa; mucosal atrophy & n/a & 8 & n/a & $n / a$ & $n / a$ \\
\hline 7 & Nodule in antrum, erythematous mucosa in body & Antrum type gastric mucosa with mild chronic inflammation & 7 & $n / a$ & $n / a$ & $\mathrm{n} / \mathrm{a}$ \\
\hline 8 & Erythematous mucosa, body & n/a & 5 & n/a & $n / a$ & n/a \\
\hline 9 & Normal & $n / a$ & 6 & $n / a$ & $n / a$ & $n / a$ \\
\hline 10 & Nodular mucosa in fundus and body, friable mucosa & n/a & 7 & n/a & $n / a$ & Body (greater curvature)/ 0.3 \\
\hline 11 & Polyps & $n / a$ & 6 & n/a & n/a & Upper fundus/0.9 \\
\hline 12 & Single polyp & Fundic gland polyp & 6 & n/a & $n / a$ & Fundus $/ 2.0$ \\
\hline 13 & Erythematous mucosa, single papule & Gastric antral mucosa with no pathological changes & 11 & $n / a$ & $n / a$ & $\mathrm{n} / \mathrm{a}$ \\
\hline 14 & Normal & n/a & 9 & n/a & $n / a$ & n/a \\
\hline 15 & Single polyp & Fundic gland polyp & 10 & $\mathrm{n} / \mathrm{a}$ & $\mathrm{n} / \mathrm{a}$ & $\mathrm{n} / \mathrm{a}$ \\
\hline 16 & Mucosal atrophy; erythematous mucosa in antrum, single polyp & Oxyntic mucosa with a few dilated glands, possibly a fundic gland polyp & 8 & n/a & $n / a$ & n/a \\
\hline 17 & Enythematous mucosa in antrum, possible angioectasia & $n / a$ & 10 & $\mathrm{n} / \mathrm{a}$ & $\mathrm{n} / \mathrm{a}$ & $\mathrm{n} / \mathrm{a}$ \\
\hline 18 & Enthematous mucosa in antrum, possible angioectasia & $\mathrm{n} / \mathrm{a}$ & 10 & n/a & $n / a$ & n/a \\
\hline 19 & Friable mucosa & Gastric body mucosa with no specific pathology & 8 & $n / a$ & $\mathrm{n} / \mathrm{a}$ & $\mathrm{n} / \mathrm{a}$ \\
\hline 20 & Normal stomach & $\mathrm{n} / \mathrm{a}$ & 6 & n/a & $n / a$ & Body (lesser curvature)/0.4 \\
\hline 21 & Submucosal tumor with central umbilication in antrum & Antral mucosa, no pathologic changes & 9 & $\mathrm{n} / \mathrm{a}$ & $\mathrm{n} / \mathrm{a}$ & Body (greater curvature/0.1 \\
\hline 22 & Multiple hyperplastic polyps, erythematous mucosa in fundus & Gastric oxyntic mucosa with mild chronic gastritis with increased eosinophils & 6 & n/a & $n / a$ & n/a \\
\hline 23 & Polyps & Fundic gland polyp & 11 & $\mathrm{n} / \mathrm{a}$ & $\mathrm{n} / \mathrm{a}$ & $\mathrm{n} / \mathrm{a}$ \\
\hline 24 & Friable, nodular mucosa & Chronic active gastrtitis (Helicobacter pylori positive by immunostain) & 13 & n/a & $\mathrm{n} / \mathrm{a}$ & Body/1.0 \\
\hline 25 & Nodular mucosa in fundus and body; polyps, small white spots & Mild chronic gastritis & 7 & $\mathrm{n} / \mathrm{a}$ & $\mathrm{n} / \mathrm{a}$ & $\mathrm{n} / \mathrm{a}$ \\
\hline 26 & Solitary pale spot in fundus & Oxyntic mucosa with no pathologic changes & 7 & n/a & n/a & n/a \\
\hline 27 & Normal & $\mathrm{n} / \mathrm{a}$ & 7 & Posterior fundus, anterior upper middle body/0.1 and 0.9 & $\mathrm{n} / \mathrm{a}$ & Upper fundus/2.0 \\
\hline 28 & Nonbleeding erosive gastropathy & Oxyntic and antral type gastric mucosa with focal chronic inflammation & 7 & Posterior upper middle body, posterior fundus/ 0.5 and 0.2 & Fundus/ 0.7 & $n / a$ \\
\hline 29 & Polyps & $n / a$ & 8 & Anterior fundus/ 0.4 and 0.7 & $\mathrm{n} / \mathrm{a}$ & Cardia, fundus (anterior + posterior wall, Body (anterior + posterior wall, lesser + greater curvature)/4.0 \\
\hline 30 & Normal & n/a & 9 & n/a & $\mathrm{n} / \mathrm{a}$ & Upper fundus $/ 0.8$ \\
\hline 31 & Single papule in antrum; multiple papules in fundus; erythematous mucosa & Antral mucosa with mild chronic gastritis, oxyntic mucosa with mild chronic gastritits & 1 & n/a & $\mathrm{n} / \mathrm{a}$ & n/a \\
\hline 32 & Normal & n/a & 12 & $\mathrm{n} / \mathrm{a}$ & n/a & n/a \\
\hline 33 & Eythematous mucosa in antrum & $\mathrm{n} / \mathrm{a}$ & 8 & n/a & $n / a$ & n/a \\
\hline 34 & Gastritis, single papule & Gastric body type mucosa with no pathological changes & 6 & Anterior fundus $/ 0.3$ & $n / a$ & Fundus, body / 2.0 \\
\hline 35 & Friable mucosa & Chronic gastritis & 6 & $n / a$ & $n / a$ & Body (greater curvature)/<1.0 \\
\hline 36 & Few papules; enthematous mucosa in antrum; nodular mucosa in body & Gastric mucosa with hyperplastic foveolar gland and reactive changes & 12 & $n / a$ & n/a & $n / a$ \\
\hline
\end{tabular}

